\title{
Electron Capture Dissociation Product Ion Abundances at the $X$ Amino Acid in RAAAA-X-AAAAK Peptides Correlate with Amino Acid Polarity and Radical Stability
}

\author{
Aleksey Vorobyev, Hisham Ben Hamidane, and Yury O. Tsybin \\ Biomolecular Mass Spectrometry Laboratory, Ecole Polytechnique Fédérale de Lausanne, Lausanne, \\ Switzerland
}

\begin{abstract}
We present mechanistic studies aimed at improving the understanding of the product ion formation rules in electron capture dissociation (ECD) of peptides and proteins in Fourier transform ion cyclotron resonance mass spectrometry. In particular, we attempted to quantify the recently reported general correlation of ECD product ion abundance (PIA) with amino acid hydrophobicity. The results obtained on a series of model H-RAAAAXAAAAK$\mathrm{OH}$ peptides confirm a direct correlation of ECD PIA with $\mathrm{X}$ amino acid hydrophobicity and polarity. The correlation factor (R) exceeds 0.9 for 12 amino acids (Ile, Val, His, Asn, Asp, Glu, Gln, Ser, Thr, Gly, Cys, and Ala). The deviation of ECD PIA for seven outliers (Pro is not taken into consideration) is explained by their specific radical stabilization properties (Phe, Trp, Tyr, Met, and Leu) and amino acid basicity (Lys, Arg). Phosphorylation of Ser, Thr, and Tyr decreases the efficiency of ECD around phosphorylated residues, as expected. The systematic arrangement of amino acids reported here indicates a possible route toward development of a predictive model for quantitative electron capture/transfer dissociation tandem mass spectrometry, with possible applications in proteomics. (J Am Soc Mass Spectrom 2009, 20, 2273-2283) Published by Elsevier Inc. on behalf of American Society for Mass Spectrometry
\end{abstract}

$\mathrm{R}$ evealing peptide and protein structure-activity and structure-function relationships is an important and challenging step in the drug discovery process. Further insights into the influence of specific amino acids on molecular conformation and physicochemical properties are needed to increase the throughput and accuracy of the methods and techniques used to investigate these critical relationships [1, 2]. Electron capture dissociation (ECD) [3] and electron-transfer dissociation (ETD) [4,5] are generally employed to determine peptide and protein primary structures and to characterize labile modifications in tandem mass spectrometry (MS/MS)-based proteomics. In addition, recent findings demonstrate that the probability of a given $\mathrm{N}-\mathrm{C}_{\alpha}$ bond in a peptide backbone being cleaved by ECD/ETD, which can be monitored by product ion abundance (PIA), is governed by peptide or protein conformation [6-9]. A number of experiments ranging from targeted peptide analysis to statistical interpretation of fragmentation have been performed for several thousand tryptic peptides $[6,7,10-13]$ to examine the dependence of ECD PIA on peptide sequence. The experimental results have confirmed the complementarity of ECD and vibrationally induced dissociation, e.g.,

Address reprint requests to Professor Y. O. Tsybin, Biomolecular Mass Spectrometry Laboratory, Ecole Polytechnique Fédérale de Lausanne, BCH 4307, 1015 Lausanne, Switzerland. E-mail: yury.tsybin@epfl.ch collision-induced dissociation (CID), but have failed to provide a general quantitative model for ECD. Enhancing models to include intramolecular hydrogen bonding has provided the best quantitative description of ECD PIA to date, as shown by Zubarev and coworkers [14]. However, their reported correlation is built upon extensive molecular dynamics simulations performed for a specific case of a selected part of a small (20-amino acid) protein, Trp cage. Although general application of the model would be time-consuming, it demonstrates the importance of hydrogen bonds when attempting to quantitatively describe ECD. Recent molecular dynamics modeling complemented with quantum chemistry calculations on penta- and hexapeptides supports the hypothesis that ECD/ETD possess strong conformational selectivity [15]. The reported results demonstrate the ability to find specific peptide conformations that lead to formation of a given product ion in ECD or ETD mass spectra. However, the extremely large size of a peptide's conformational space and the fact that the same product ion can be formed from a number of conformers via different pathways limits the ability to quantitatively model ECD/ETD fragmentation patterns. Importantly, the performed calculations do not take into account peptide properties that influence peptide conformation and dynamics, e.g., entropy and ion internal energy $[16,17]$. Therefore, an alternative, presumably phe- 
nomenological model may be helpful when developing a quantitative description of ECD/ETD.

Recently, we reported observation of sequencedependent ECD PIA of amphipathic peptides [12]. Due to periodic variation of spatially separated hydrophobic and hydrophilic sequence regions in amphipathic peptides, their most probable conformational state in solution is an $\alpha$-helix. Distinguishing sequence and conformational (e.g., secondary structure) effects on ECD PIA of amphipathic peptides is a complex task requiring extensive computational and experimental studies. Nevertheless, our observed correlation of ECD PIA with the amino acid hydrophobicity distribution represents a novel insight into the dependence of ECD on peptide sequence. Therefore, we have taken the suggested correlation between ECD PIA and amino acid hydrophobicity as a starting point in developing a quantitative phenomenological description of ECD/ETD.

Here we present a mechanistic study on the influence of amino acid properties, particularly hydrophobicity/polarity and radical stability, on ECD PIA. ECD Fourier transform ion cyclotron resonance mass spectrometry (FT-ICR MS) patterns of a complete series of model, single amino acid-substituted H-RAAAAXAAAAK$\mathrm{OH}$ peptides involving all 20 naturally occurring and three phosphorylated amino acids are compared and correlated with various $X$ amino acid physicochemical properties. The presented results can be considered an initial step toward development of a quantitative ECD/ETD model.

\section{Experimental}

\section{Design and Synthesis of Model Peptides}

A series of model peptides based on the general sequence H-RAAAAXAAAAK-OH (where $\mathrm{X}$ is one of 20 natural amino acids or a phosphorylated T, Y or S) was produced by solid-state Fmoc chemistry on an Applied Biosystems 433 A Synthesizer with further purification by liquid chromatography (Protein and Peptide Synthesis Facility, Biochemistry Department, University of Lausanne, Switzerland). Peptides were dissolved in water to a concentration of $\sim 1 \mathrm{mM}$ and further diluted in a standard spraying solution $\left(\mathrm{H}_{2} \mathrm{O} / \mathrm{CH}_{3} \mathrm{OH}\right.$ 50:50 volume ratio with $1 \% \mathrm{H}_{3} \mathrm{COOH}$ ) to a final peptide concentration of $\sim 1 \mu \mathrm{M}$. Circular dichroism (CD) spectroscopy measurements were performed at $25^{\circ} \mathrm{C}$ and $80{ }^{\circ} \mathrm{C}$ in either pure $\mathrm{H}_{2} \mathrm{O}$ or $50 / 50 \mathrm{H}_{2} \mathrm{O} / \mathrm{ACN}$ using a J-810 CD system from Jasco (St. Michaels, MD, USA) equipped with a $500-\mu \mathrm{L}$ cell.

The design of model peptides to probe the effect of amino acid properties on ECD PIA was performed based on ECD-specific experimental constraints (e.g., influence of charge location on ECD PIA) and custom structures of model peptides employed in amino acid hydrophobicity scale analysis (e.g., the translocon insertion method). Scheme 1 illustrates the structure of H-RAAAAXAAAAK-OH peptides with an exchangeable middle amino acid. The N-terminal Arg and C-terminal Lys define charge location and provide sufficient ionization efficiency for multiply charged ion production. Although the N-terminal amide can compete with Arg and Lys for a proton, we believe that the side-chains of the basic amino acids located next to the termini were the primary locations of protonation. We confirmed proton location on Arg and Lys in peptides without a basic amino acid (e.g., Lys, Arg, and His) in the middle by joint theoretical peptide conformation landscaping with replica exchange molecular dynamics simulations and an experimental ion mobility mass spectrometry-based ap-

\section{modification}

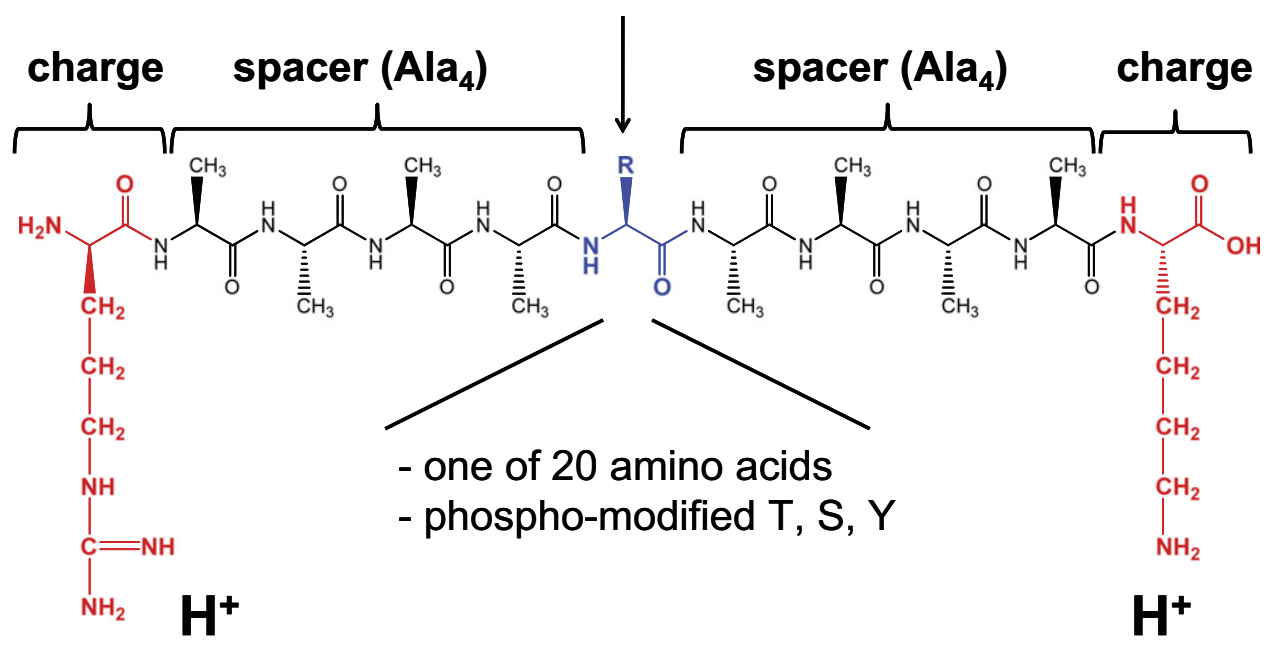

Scheme 1. Model peptides designed to probe the effect of a single amino acid substitution on product ion abundance in ECD. Polyalanine spacers separate protonated N-terminal and C-terminal basic amino acids from a centrally located modification. All 20 naturally occurring amino acid residues have been examined, as have phosphorylated Tyr, Ser, and Thr. 
proach [18]. The substantial difference in mass between Lys and Arg side chains separates potentially overlapping C-terminal and N-terminal product ions of poly-Ala peptides. (Ala) $)_{4}$ spacers were designed to be long enough to keep a middle amino acid of interest far enough away from the protonated sites to reduce the potential for direct charge influence. On the other hand, spacers longer than four amino acids may substantially reduce ECD efficiency in the central part of the sequence for doubly charged precursor ions [12]. The model peptide with $X=$ Ala may be considered as a reference molecule without a structural "defect" at the central amino acid.

\section{ECD-Based Tandem Mass Spectrometry}

ECD experiments were performed on a hybrid linear ion trap Fourier transform ion cyclotron resonance mass spectrometer (LTQ FT-ICR MS) equipped with an 11 T superconducting magnet [12, 19]. Peptides were electrosprayionized with a TriVersa robot using a standard ESI chip (10 $\mu \mathrm{m}$ i.d. capillaries) from Advion (Ithaca, NY, USA) at a flow rate of $\sim 200 \mathrm{~nL} / \mathrm{min}$. Doubly protonated peptides were isolated in the LTQ (isolation window of $4 \mathrm{Th}$ ) and transferred to the ICR ion trap for subsequent tandem mass spectrometry following standard procedures [20]. ECD was performed with low-energy electrons for $\sim 70 \mathrm{~ms}$ using a pencil electron beam from a dispenser cathode located in the homogenous region of the magnetic field [21]. The variable delay before electron injection was optimized to account for ion magnetron motion [22]. To improve the statistical validity of the acquired data, 100 single-scan mass spectra were summed to produce each mass spectrum. ECD PIA analysis was performed as previously described [12]. Briefly, isotopic peaks for each product ion were summed to provide a list of absolute abundances for product ions in a given tandem mass spectrum. Relative values for the ECD PIA were then obtained by dividing the absolute values of $c$-type or $z$-type product ions by the sum of abundances for all c-type ion series and $z$-type ion series. Taking into account all isotopes for a given product ion diminishes the discrepancy in ion internal energies between different experiments as isotopes of both odd-electron (radical) and even-electron (prime) product ions are considered [23]. Statistical analysis of the relative ECD PIA variation within a single accumulated spectrum (100 summed scans) gives an estimated error of less than $1 \%$. The error in repeatability and reproducibility of relative ECD PIA is more pronounced, with an estimated $5 \%$ to $10 \%$ day-to-day error for replicate experiments performed on the same instrumental set-up. The variation is presumably due to the influence of precursor ion and incoming electron energy sensitivity to experimental parameters during the ECD process. Despite the use of automatic gain control (AGC), electrospray instabilities modulate the amount of precursor ions in the ICR trap, thereby influencing the ion-electron beam overlap and the am- plitude of space charge effects. Additional error is due to imperfect product ion excitation and detection repeatability. Nevertheless, local maxima and minima of the reported ECD PIA distributions are reproducible even with the estimated errors.

\section{Results and Discussions}

\section{ECD PIA of H-RAAAAXAAAAK-OH Peptide Series}

ECD FT-ICR mass spectra of the model peptide series demonstrate extensive sequence coverage by primarily c-type ions for all peptides studied (Figure 1 and Supplemental Material Figure S1, which can be found in the electronic version of this article). Although several $c$-ions show radical components, yield of oddelectron $c$-ions is low. ECD PIA distributions show a characteristic response to the "defect" (e.g., amino acid substitution) incorporated into the polyAla chain, as shown in Figure 2. The dominance of $c$-type ions is presumably due to higher propensity of Lys compared with Arg for charge neutralization in ECD [24]. Nevertheless, $z$-type ions formed by $\mathrm{N}-\mathrm{C}_{\alpha}$ cleavage near the $\mathrm{N}$-terminus were observed for all peptides. Additionally, several peptides demonstrate formation of $z$-ions around the substituted amino acid. To study the impact of the substituted amino acid on ECD PIA, difference graphs were constructed. The difference graphs (Figure 3) demonstrate the variation of ECD PIA versus a polyAla chain (e.g., the peptide where $X=$ Ala, also referred to as the reference peptide). ECD of the reference peptide demonstrates almost equal probability for the $\mathrm{N}-\mathrm{C}_{\alpha}$ bond cleavage in the middle of the sequence, with decreasing bond rupture efficiency toward the $\mathrm{N}$-terminus. The $\mathrm{N}$-terminus remains charged during $c$-ion formation. A line is included at a value of zero change in the ECD PIA shown in Figure 3 to help highlight up- and down-regulation of the ECD PIA when compared with the $X=$ Ala peptide. Amino acids in Figure 3 are grouped in regard to the fragmentation behavior they demonstrate. As shown in Figure $3 \mathrm{~d}-\mathrm{f}$, enhanced fragmentation generally occurs at the Cterminal side of the inserted amino acid (at $\mathrm{N}-\mathrm{C}_{\alpha}$ bond number 7). In contrast, ECD efficiency is enhanced at the N-terminal side of the inserted amino acid (e.g., $\mathrm{N}-\mathrm{C}_{\alpha}$ bond number 6) for Met and Leu residues (Figure 3c). Ile and Val, the most hydrophobic amino acids, demonstrate enhanced ECD efficiency at $\mathrm{N}-\mathrm{C}_{\alpha}$ bond number 5, even further toward the N-terminus. A symmetric decline in ECD PIA at $\mathrm{N}-\mathrm{C}_{\alpha}$ bond number 6 is observed for Ile and Val. Of the 3 basic amino acids, His demonstrates ECD PIA behavior similar to polar residues (e.g., Glu). Substitution with more basic Arg and Lys (Figure 3b) yields drastic differences in the ECD PIA distribution along the sequence. Presumably, His is not charged in the $X=$ His peptide due to the higher basicities of the Arg and Lys residues at the 


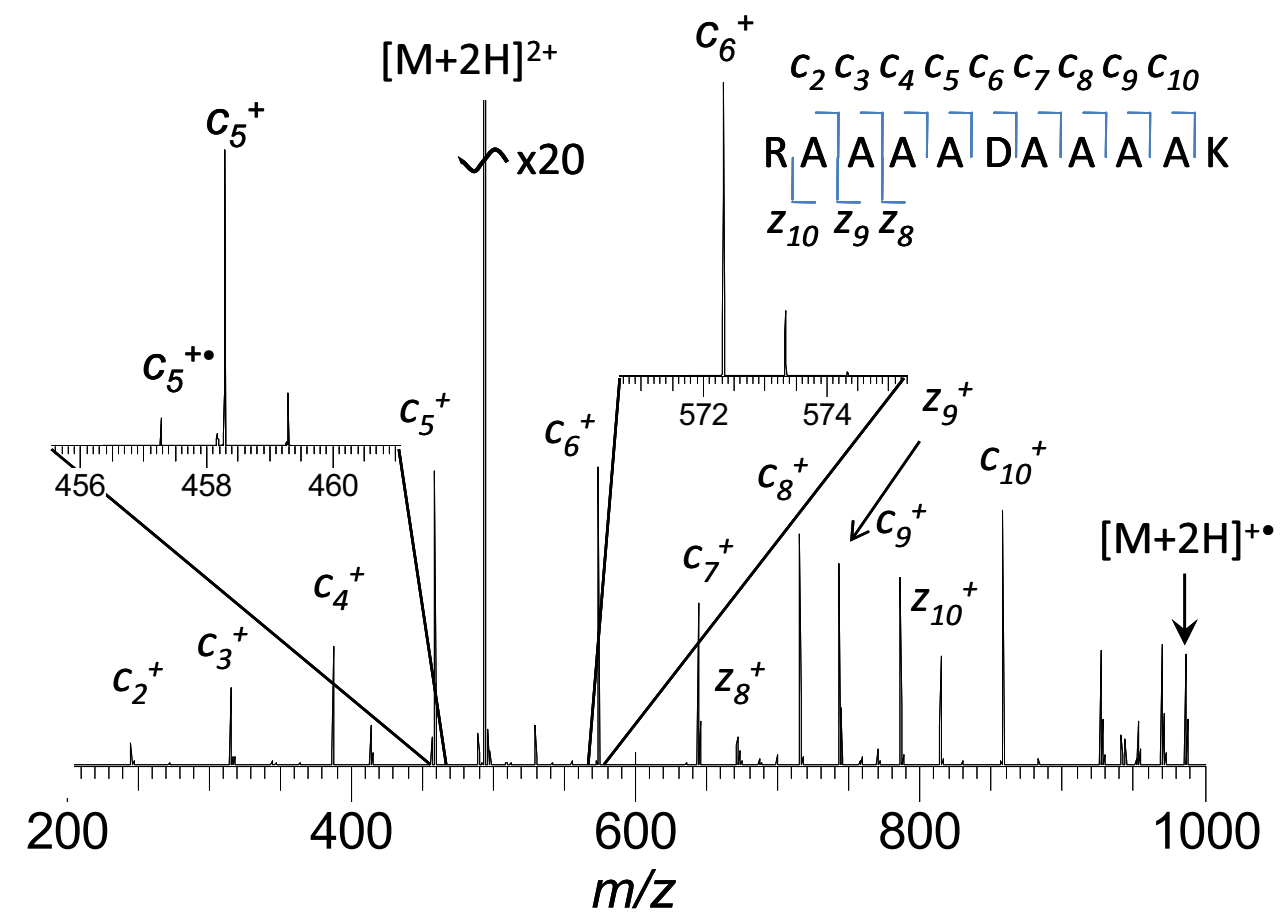

Figure 1. A typical tandem mass spectrum of peptide H-RAAAADAAAAK-OH, acquired under standard ECD FT-ICR MS conditions. Data for all other peptides are shown in the Supplementary material (Figure S1).

peptide termini, and thus His does not substantially impact charge location.

The secondary structure of the H-RAAAAXAAAAK$\mathrm{OH}$ peptides were probed by circular dichroism (data not shown). No pronounced secondary structure elements, e.g., an $\alpha$-helix or a $\beta$-strand, could be assigned to any of the peptides in the series.

\section{Correlation of ECD PIA with Amino Acid Properties}

We sought to determine the particular amino acid properties that influence the ECD PIA through correlation of amino acid hydrophobicity or polarity scales with the ECD PIA around the substituted amino acid, following recent reports of a correlation between the ECD PIA and the amino acid hydrophobicity [12]. However, to distinguish the influence of hydrophobicity/polarity on ECD fragmentation pattern from other amino acid properties, we had to exclude a number of amino acids from the consideration. First, based on the previous knowledge on the influence of basic residues on peptide protonation and ECD fragmentation pattern, we excluded the basic amino acids (Lys and Arg) from the correlation fit. The data reported in Figures 2 and 3 clearly suggest charge location perturbation within the precursor ions for $X=\operatorname{Arg}$ and $X=$ Lys. Second, we excluded the group of five amino acids, namely Leu, Phe, Tyr, Trp, and Met, which are known to provide strong radical stabilization that may dominate hydrogenbonding properties reflected by hydrophobicity or po- larity $[25,26]$. Radical stabilization in molecular system depends on the possibility of delocalization of the electron density excess on as many atoms or groups of atoms as possible to minimize its potential energy. To evaluate the extent of stabilization, two criteria should be taken into consideration: the degree of bonding of the atom on which the radical is initially located, and the proximity of stabilizing groups (such as electron withdrawing groups). As ECD and ETD are considered to be radical-driven, the extent of radical stabilization should play a crucial role in determining the Gibbs enthalpy of the reaction and the relative energy of the resulting product ions. A reasonable assumption is that to form fragment ions, namely $c$ and $z^{*}$, not only the activation barrier leading to the $\mathrm{N}-\mathrm{C}_{\alpha}$ bond cleavage has to be overcome but also the resulting radical has to be stable. From that perspective, leucine should provide higher radical stability than isoleucine despite its comparable hydrophobicity/polarity values. That is because leucine radical is stabilized on a tertiary carbon atom, whereas it is stabilized only on a secondary or a primary carbon for isoleucine. Substantial difference between impact of Leu and Ile on radical-driven peptide fragmentation was recently confirmed by Julian and coworkers [25]. Similarly, although methionine and cysteine are close on a hydrophobicity/polarity scale, methionine is characterized by stronger radical stabilizing properties than cysteine. Methionine may form secondary sulfur radical upon ECD, enhancing product ion stabilization and decreasing its energy, whereas cysteine forms only primary sulfur radical that does not 

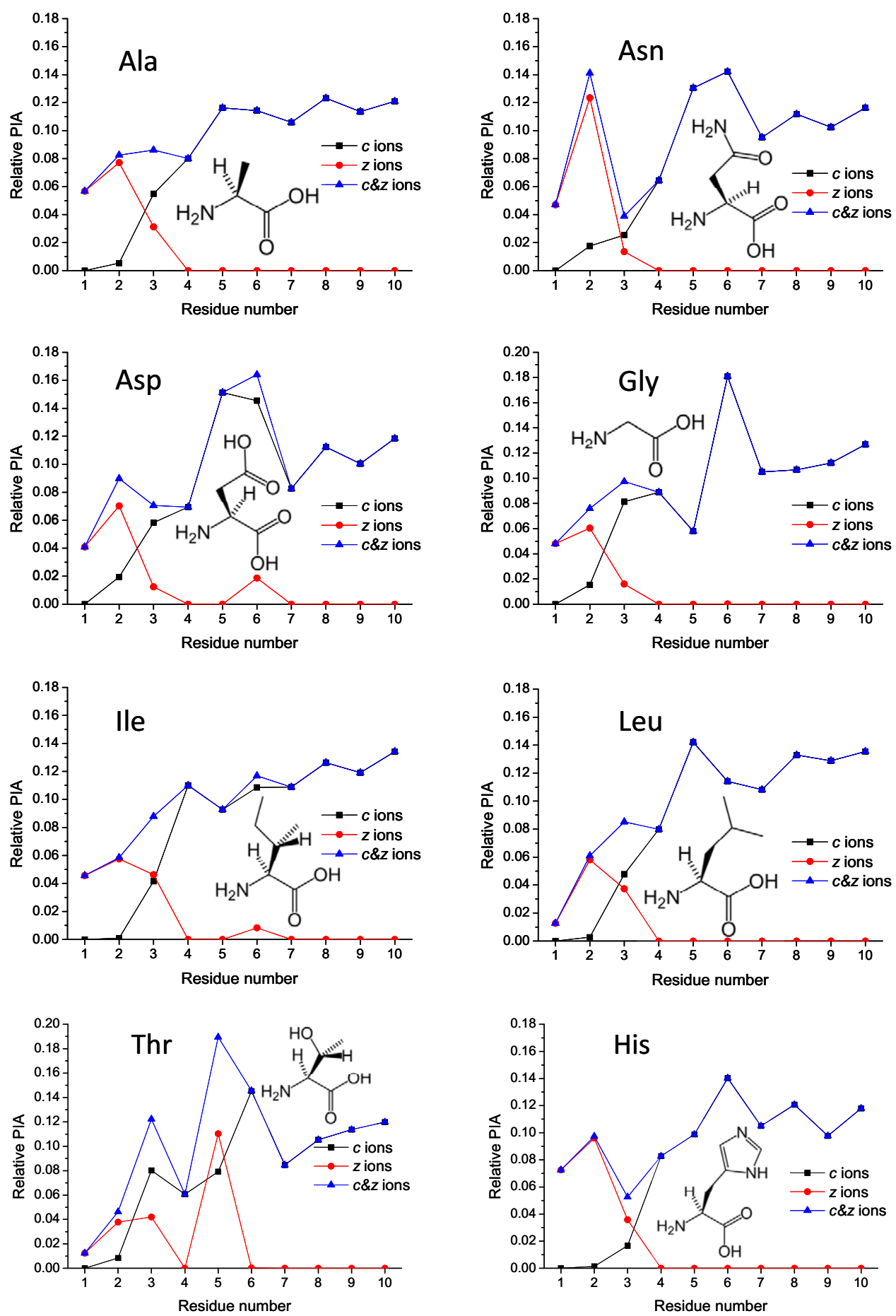

Figure 2. Product ion abundance distribution for $c$-ions, $z$-ions, and a sum of $c$ - and $z$-ions obtained by ECD FT-ICR MS of peptides H-RAAAA-X-AAAAK-OH, where $\mathrm{X}$ is one of 20 naturally occurring amino acids. Product ion abundances are normalized to the sum of all $c$ - and $z$-ions. The statistical error of the relative ECD PIA variation is less than $1 \%$. C-terminal product ions ( $z$-ions) are shown in inverse order to correlate with the corresponding $c$-ions.

provide extensive radical stabilization. The remaining amino acids, Phe, Tyr, and Trp, are aromatic and, thus, provide strong radical stabilization due to their specific ring-associated radical-forming properties.

With the exception of five amino acids, which are excluded due to their known radical-stabilizing prop- erties and two basic amino acids, excellent correlation is observed between the ECD PIA and the hydrophobicity and polarity of the substituted residue, Figure 4 . The top of Figure 4 demonstrates a correlation of the ECD PIA with Hessa's amino acid hydrophobicity scale, employed in [12]. The bottom of Figure 4 shows the 

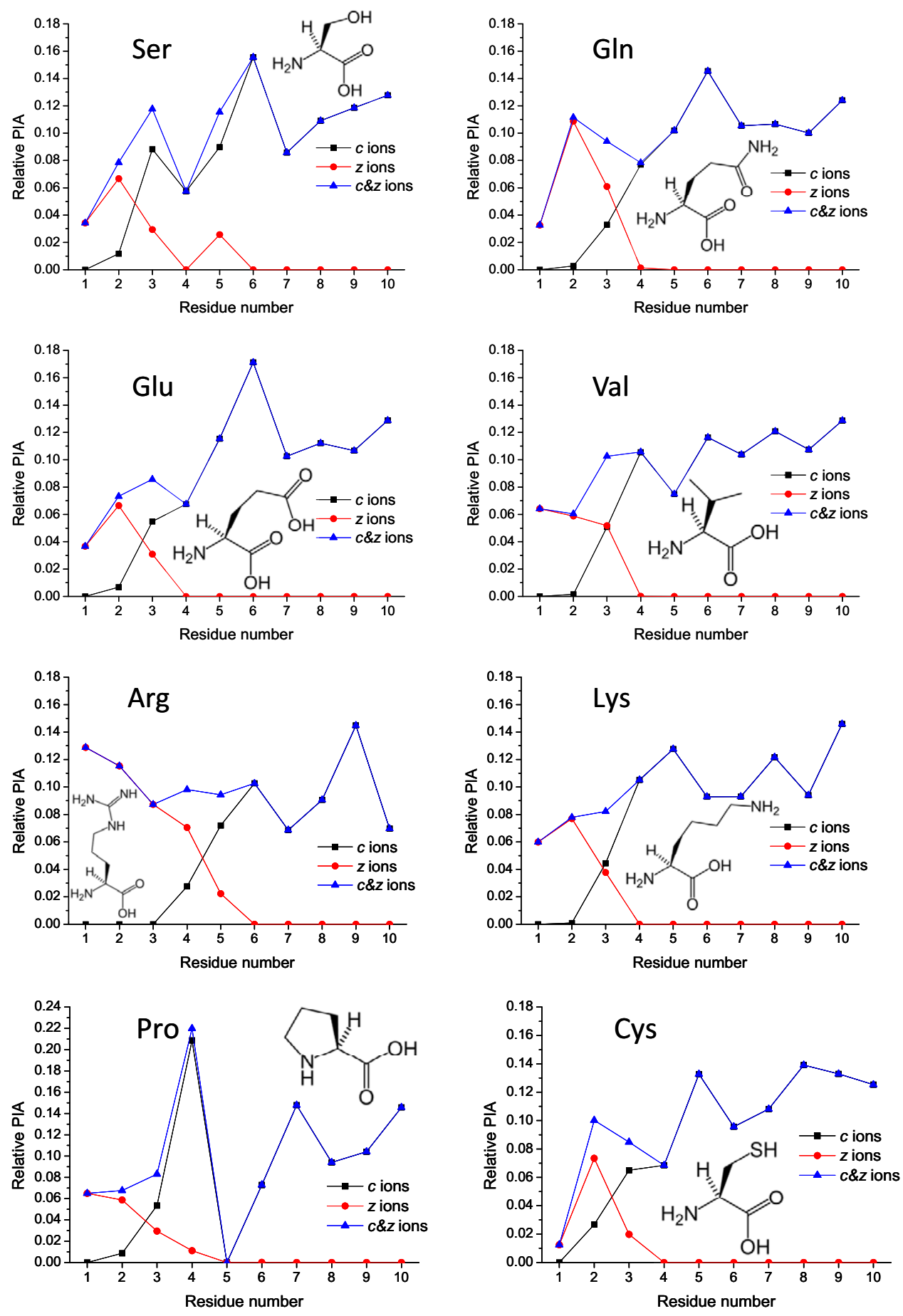

Figure 2. Continued.

ECD PIA plotted versus the amino acid polarity scale introduced by Grantham [27]. A linear fit shows almost direct correlation between the ECD PIA and amino acid polarity with a correlation factor of $\mathrm{R}=0.97$ for the remaining 12 amino acids (Figure 4, bottom). The ECD PIA correlation with the Hessa amino acid hydrophobicity obtained for the same 12 amino acids demonstrates a slightly lower correlation factor of $R=0.93$ (Figure 4, top). The direct correlation reported here includes more amino acids and achieves a higher correlation factor than previously reported correlations of cleavage frequencies in ECD versus amino acid properties. The initial assumption that the ECD PIA correlates with amino acid hydrophobicity has been confirmed for most of the amino acids.

We consider polarity scales here due to the direct relation of polarity to hydrophobicity, with polarity defined in a similar manner but for a broader range of 

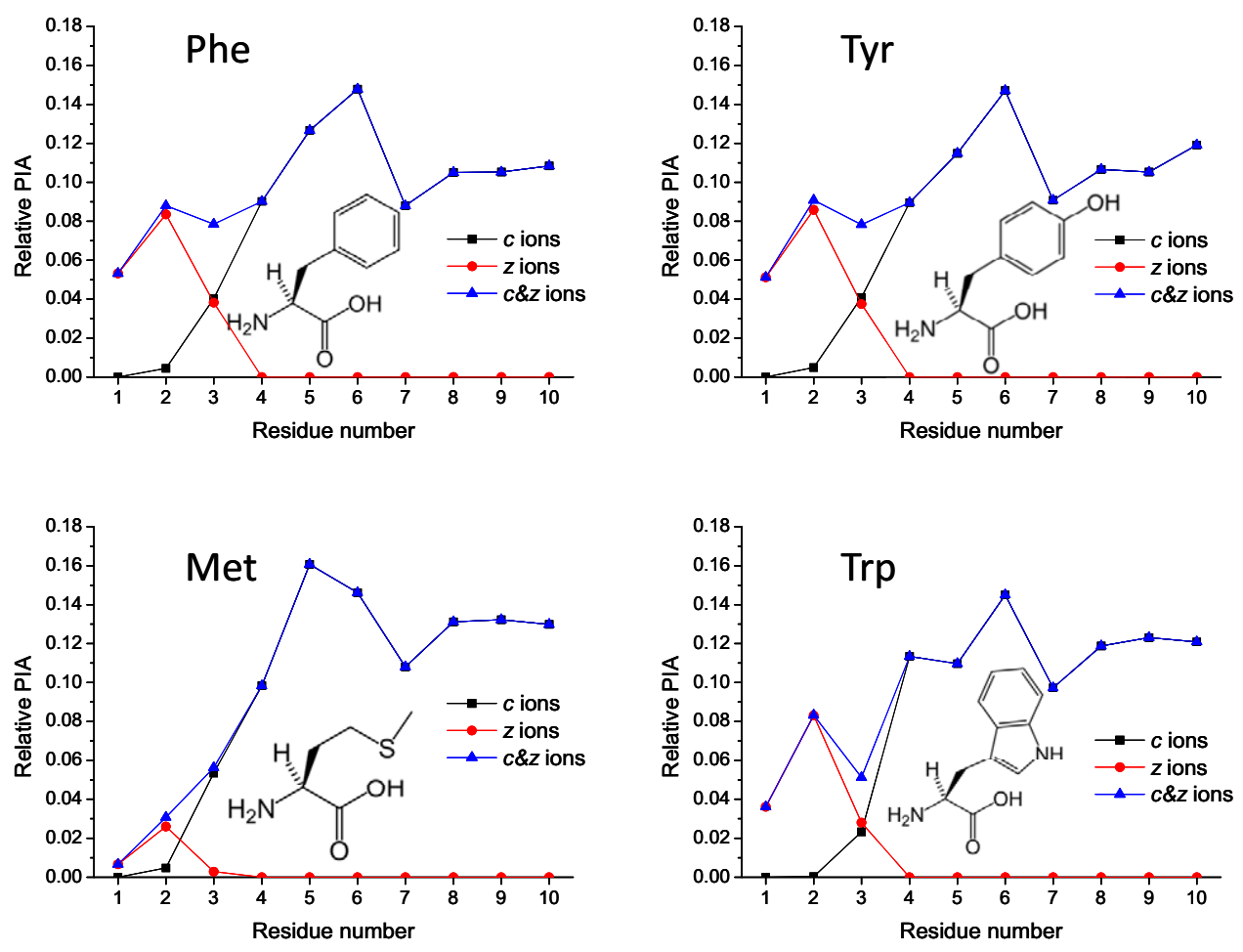

Figure 2. Continued.

solvents than hydrophobicity. This particular scale of polarity is selected because it correlates best with the ECD PIA for most amino acids as described below. Only c-ions around the substituted amino acids are taken into account in Figure 4. Normalization is done on the sum of all $c$-ions, with the $z$-ions excluded from consideration. Interestingly, including $z$-ions into normalization slightly reduces the overall correlation ( $R$ $\sim 0.8$ ). Indeed, data in Figures 2 and 3 clearly indicate absence of $z$-ions for the $\mathrm{N}-\mathrm{C}_{\alpha}$ cleavages around the middle amino acid in most cases with the exception for Asp, Ile, Ser, Arg, and Thr. The most abundant $z$-ion is observed for Thr despite its lower hydrophobicity/ polarity compared with other amino acids from this group, Figure 2. Although previously reported data confirm correlation of $z$-ion abundance with amino acid hydrophobicity for ECD of doubly and triply charged peptides [12], data presented here indicate different reactivity of $z$-ions compared with the $c$-ions, presumably due to their radical nature. Furthermore, for the doubly charged peptide series employed in the current work, $z$-ions could only be formed accompanied by the charge neutralization at the N-terminal Arg. On the other hand, $c$-ions could only be formed accompanied by the charge neutralization at the C-terminal Lys. Therefore, differences in peptide conformations due to the interactions between Arg and Lys side chains with a middle amino acid side chain may also contribute to the observed behavior. Understanding the selectivity and specificity of ECD to the amino acid nature and peptide properties in the formation of $z$-ions requires further research.
Although incorporation of basic amino acids (Lys and Arg) drastically changes the ECD PIA distribution compared with non-basic amino acids, Figures 2 and 3, the ECD PIA for Arg is close to the main trend line, Figure 4. We consider the location of Arg on the main trend to be a coincidence, as the fragmentation efficiency around the $\mathrm{N}$-terminus and $\mathrm{C}$-terminus in the ECD of those peptides with $X=\operatorname{Arg}$ is perturbed. The reported data, however, do not allow estimation of the probability that the middle amino acid is protonated when $X=$ Lys or Arg. Complementary experimental measurements (e.g., ion mobility mass spectrometry) combined with molecular dynamics simulations are needed. Importantly, all five amino acids that provide strong radical stabilization cluster outside the linear fit. They demonstrate higher ECD efficiency for the backbone cleavages around them, as expected, Figure 4.

Table 1 summarizes both the reported values for the ECD PIA around substituted amino acids and the corresponding properties of the amino acids. The proline residue is not taken into account when examining the general correlation. Table 1 also shows that phosphorylation of a middle site in model peptides with $\mathrm{X}=$ Thr, Tyr, and Ser reduces the likelihood that $\mathrm{N}-\mathrm{C}_{\alpha}$ bonds located close to the modification will be cleaved. This decrease in the ECD efficiency is in agreement with the recent studies by Creese and Cooper, which suggest reduction in ECD efficiency of phosphopeptides due to the formation of salt-bridges between the phosphorgroups and protonated amino acids [28]. Furthermore, phosphorylation reduces radical stabilization of tyrosine [29]. Finally, phosphor groups may act as radical 

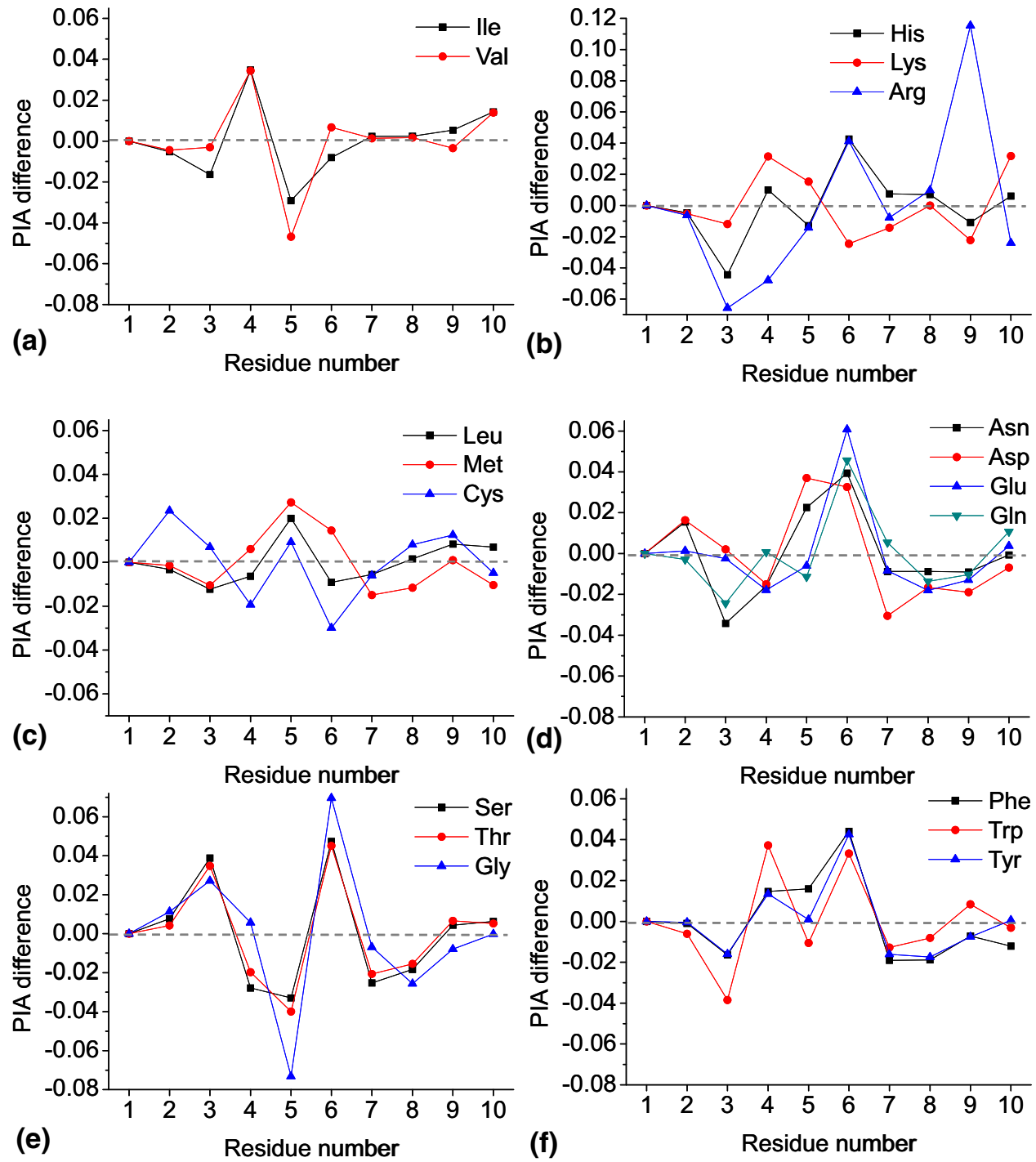

Figure 3. Difference graphs for product ion abundance distribution versus $X=$ Ala for $c$-ions obtained by ECD FT-ICR MS of H-RAAAA-X-AAAAK-OH, where X is (a) Ile and Val; (b) His, Lys, and Arg; (c) Leu, Met, and Cys; (d) Asn, Asp, Glu, and Gln; (e) Ser, Thr, and Gly; (f) Phe, Trp, and Tyr. The statistical error of the relative ECD PIA variation is less than $1 \%$.

traps and inhibit radical-driven fragmentation reaction [30]. Therefore, despite the liquid chromatographymass spectrometry-based experimental fact that phosphorylation increases overall peptide hydrophilicity, other processes prohibit increase of ECD efficiency.

In total, a set of 17 amino acid property scales out of the 60 probed [31] returned a correlation factor $R$ higher than 0.5 for all amino acids but Arg, Lys, His, Gly, Pro, Cys, and Leu. Among others, the following scales were considered: hydrophobicity (Hessa [32], Kyte and Doolittle [33], Black [34]), polarity (Grantham [27], and Zimmerman et al. [35]), $\alpha$-helix tendency [36], $\beta$-turn tendency [36], and transmembrane tendency [37]. In addition, we correlated other physicochemical properties of amino acids with the ECD PIA, including [31]: dipole moment [38], molecular weight, bulkiness [35], average flexibility index [39], pKa, substituent effect scale [40], and diverse electron properties of the amino acid side chains [41]. High correlation factors were observed when correlating the ECD PIA with the transmembrane tendency scale $(\mathrm{R}=0.89)$, hydrophobicity indices at $\mathrm{pH} 7.5$ as determined by HPLC $(\mathrm{R}=0.93)$ [42], and Black hydrophobicity $(R=0.93)$ [34]. Exclusion of amino acids from consideration was based on their specific basicity or structural properties as described above.

\section{Considerations for the ECD PIA Correlation with Polarity}

Correlation of the ECD PIA with amino acid polarity as a function of the amino acid sequence may be attributed to the ECD PIA dependence on specific conformations 

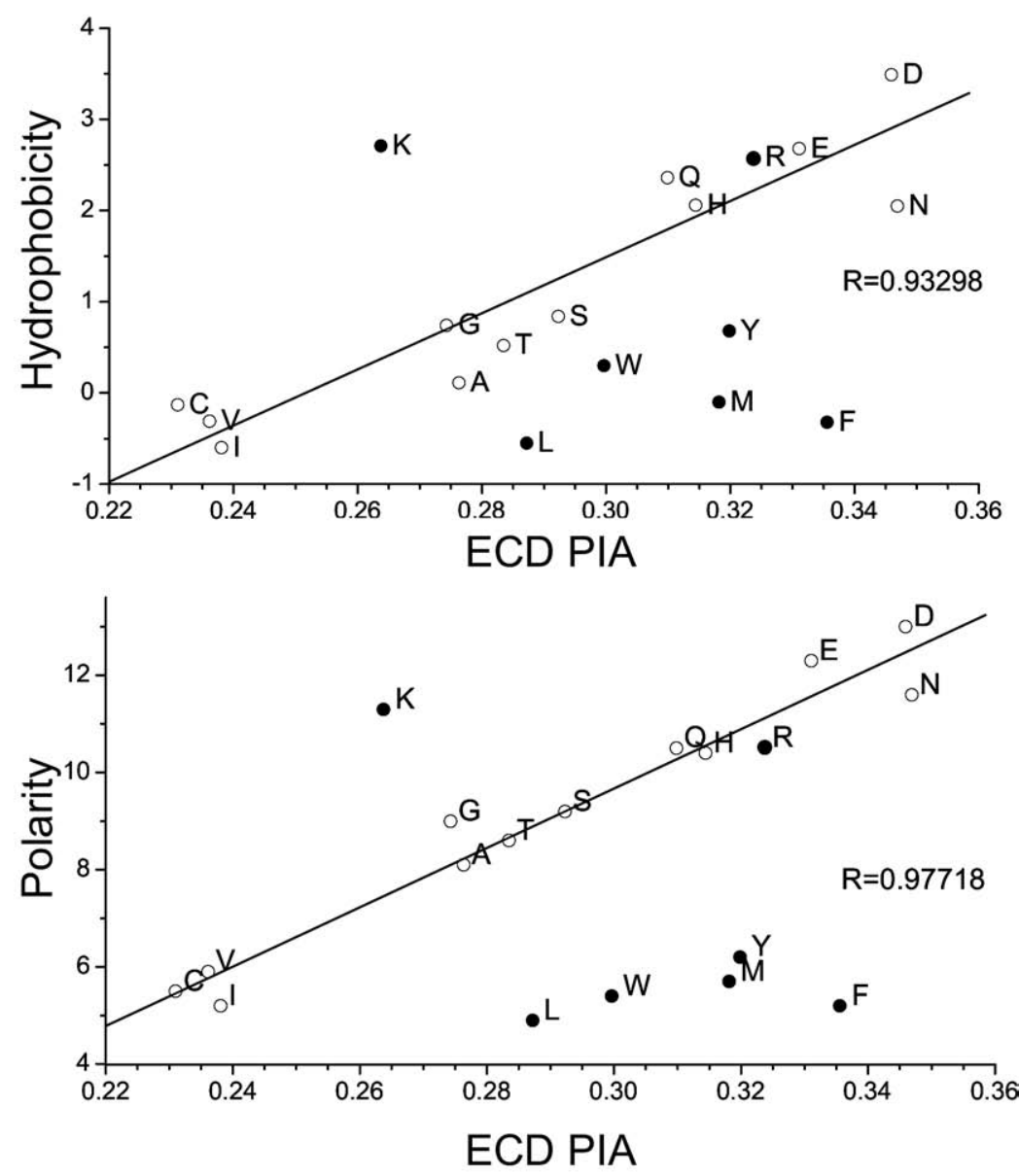

Figure 4. Correlation of the ECD product ion abundance with (top) amino acid hydrophobicity 32 and (bottom) amino acid polarity 27. The ECD product ion abundance is the summed abundance of $c$-ions due to cleavage of $\mathrm{N}-\mathrm{C}_{\alpha}$ bonds around the substituted amino acid at position $6\left(\mathrm{~N}-\mathrm{C}_{\alpha}\right.$ bonds number 6 and 7). Normalization of each ECD PIA value is performed using the sum of all detected $c$-ions. Linear correlation is performed on 12 amino acids shown by open circles. The six outliers are shown with filled circles and are not considered during correlation analysis.

that precursor ions populate. The probability of forming hydrogen bonds to and from the substituted amino acid residue in solution is presumably directly related to amino acid hydrophobicity and polarity. In addition to hydrogen bonds, the peptide conformation is influenced by other effects, including interactions between the aromatic rings or interactions between an aromatic ring and a charge. Therefore, the observed linear correlation between the ECD PIA and the amino acid hydrophobicity/polarity may indicate an amino acid-dependent peptide conformation preference and suggests that the tendency to form hydrogen bonds in the gas phase is similar to that observed in solution.

On the other hand, an alternative explanation should be entertained in which polarity is considered as a measure of electric charge separation in a peptide (e.g., the propensity to form electric dipoles). In the homogenous reference peptide with $X=A l a$, the structural "defect" is absent and the ECD PIA is not perturbed (Figure 2a). For other peptides, the middle amino acid can be considered to be a defect for energy relaxation along the peptide backbone and the hydrogen-bonded network after electron capture [43]. For instance, substituted amino acids may modulate charge (electron) transfer along the peptide's backbone and hydrogenbonding networks [44-47]. Presumably, excited electrons or "hot electrons" created at the charge neutralization site may move along the peptide structure via the electron subsystem. Indeed, the electron orbitals of atoms near the charge-solvated site are polarized before charge neutralization. Increased density of electronic states around the protonation site results in an accumulation of electrostatic energy around that point in the peptide. Upon charge neutralization, the local electric potential suddenly drops. As we recently demonstrated, acoustic phonons may be formed due to this sudden perturbation, and may distribute energy along the peptide structure upon relaxation [43]. At the same time, the electronic subsystem receives additional energy, which may lead to formation of hot, energetic electrons. Hot electrons may move in the peptide structure via covalent bonds and hydrogen bonding net- 
Table 1. Selected physicochemical properties of amino acids, e.g., hydrophobicity (after Hessa et al. 32) and polarity (after Grantham 27), versus the ECD PIA (summed abundances of $c_{5}$ ions and $c_{6}$ ions). The order is alphabetical based on the single amino acid nomenclature code. The statistical error of the relative ECD PIA variation is less than $1 \%$

\begin{tabular}{|c|c|c|c|c|}
\hline Amino acid & Hydrophobicity & Polarity & $\begin{array}{l}\text { Transm. } \\
\text { tendency }\end{array}$ & ECD PIA \\
\hline Ala (A) & 0.11 & 8.1 & 0.38 & 0.276 \\
\hline Cys (C) & -0.13 & 5.5 & -0.3 & 0.231 \\
\hline Asp (D) & 3.49 & 13 & -3.27 & 0.346 \\
\hline Glu (E) & 2.68 & 12.3 & -2.9 & 0.331 \\
\hline Phe (F) & -0.32 & 5.2 & 1.98 & 0.336 \\
\hline Gly (G) & 0.74 & 9 & -0.19 & 0.274 \\
\hline $\mathrm{His}(\mathrm{H})$ & 2.06 & 10.4 & -1.44 & 0.314 \\
\hline Ile (I) & -0.6 & 5.2 & 1.97 & 0.238 \\
\hline Lys (K) & 2.71 & 11.3 & -3.46 & 0.264 \\
\hline Leu (L) & -0.55 & 4.9 & 1.82 & 0.287 \\
\hline Met (M) & -0.1 & 5.7 & 1.4 & 0.318 \\
\hline Asn (N) & 2.05 & 11.6 & -1.62 & 0.347 \\
\hline Pro $(P)$ & 2.23 & 8 & -1.44 & 0.087 \\
\hline $\mathrm{G} \ln (\mathrm{Q})$ & 2.36 & 10.5 & -1.84 & 0.310 \\
\hline $\operatorname{Arg}(R)$ & 2.58 & 10.5 & -2.57 & 0.324 \\
\hline $\operatorname{Ser}(S)$ & 0.84 & 9.2 & -0.53 & 0.292 \\
\hline $\operatorname{Thr}(\mathrm{T})$ & 0.52 & 8.6 & -0.32 & 0.283 \\
\hline Val (V) & -0.31 & 5.9 & 1.46 & 0.236 \\
\hline $\operatorname{Trp}(\mathrm{W})$ & 0.3 & 5.4 & 1.53 & 0.300 \\
\hline $\operatorname{Tyr}(\mathrm{Y})$ & 0.68 & 6.2 & 0.49 & 0.320 \\
\hline \multicolumn{5}{|c|}{ Phosphorylated } \\
\hline $\operatorname{Ser}(p S)$ & & & & 0.202 \\
\hline $\operatorname{Thr}(p T)$ & & & & 0.163 \\
\hline Tyr(pY) & & & & 0.266 \\
\hline
\end{tabular}

works toward the remaining charge sites, e.g., toward the N-terminal Arg for the studied peptide di-cation series [44, 47]. An electron approaching the substituted amino acid detects the difference in electronic properties between Ala and the inserted amino acid. Enhanced fragmentation at the C-terminal side of the inserted amino acid (Figure $3 \mathrm{~d}-\mathrm{f}$ ) can be rationalized as a response to formation of an energetic (electrostatic) barrier for an electron to overcome. Similarly, ECD efficiency enhanced at the $\mathrm{N}$-terminal side of the inserted amino acid (Figure 3c) may indicate formation of an electrostatic well in the middle of a peptide by hydrophobic amino acids with specific radical properties. The created well would decrease the $\mathrm{N}-\mathrm{C}_{\alpha}$ bond strength inside the inserted amino acid, which corresponds to the N-terminal side of the amino acid residue. Increased amino acid hydrophobicity (Figure 3a) promotes ECD toward the N-terminus from the modification site. The probability of backbone cleavage decreases as hot electrons travel away from the charged C-terminus due to energy dissipation.

\section{Conclusions}

Correlation of the ECD PIA with amino acid hydrophobicity/polarity, a phenomenon that has recently been reported in $\mathrm{ECD}$, has been quantified for the first time using a complete amino acid-substituted set of model peptides. The high correlation factor found $(R>0.9)$ for most amino acids is somewhat surprising giving the solution phase-based definition of hydrophobicity/polarity and the gas-phase-based ECD reactions. Distinguishing three groups of amino acids (those that follow the main correlation with hydrophobicity/polarity, basic amino acids, and amino acids with strong radical stabilization preference) is less surprising, given what is known from recent literature on radical stabilization effects in ECD/ETD, and earlier reports on the influence of charge location on ECD/ETD fragmentation patterns. In accordance with the current understanding of the ECD/ETD mechanism, the reported results could be considered from two alternative directions: conformationspecific effects, and radical migration-specific effects. Both explanations require substantial theoretical calculation efforts that are currently being undertaken not only by researchers in the ECD/ETD field but also in complementary fields, e.g., radical-driven UV spectroscopy and electron photodetachment dissociation [29]. The simplicity of the model peptides selected in the current manuscript and the clear differences in the influence of different amino acids on the ECD PIA distribution may allow development of a better understanding of not only ECD, but also of other techniques in radical-driven peptide chemistry. To complete the ECD PIA data and quantum chemistry calculations, probing the peptide conformational landscape and determination of the charge location can be performed by combined ion-mobility mass spectrometry and molecular dynamics simulations [18]. Finally, the presented results indicate a possible route toward the development of a general model for predicting ECD/ETD product ion abundances in a typical proteomics experiment.

\section{Acknowledgments}

The authors thank Catherine Servis for her help during peptide synthesis, and Oleg Tsybin for discussions. They acknowledge Ecole Polytechnique Federale de Lausanne and Swiss National Science Foundation (project 200021-125147/1) for financial support.

\section{Appendix A Supplementary Material}

Supplementary material associated with this article may be found in the online version at doi:10.1016/ j.jasms.2009.08.019.

\section{References}

1. Watt, P. M. Screening for Peptide Drugs from the Natural Repertoire of Biodiverse Protein Folds. Nat. Biotechnol 2006, 24(2), 177-183.

2. Usui, K.; Tomizaki, K. Y.; Mihara, H. Screening of $\alpha$-Helical Peptide Ligands Controlling a Calcineurin-Phosphatase Activity. Bioorg. Med. Chem. Lett. 2007, 17(1), 167-171.

3. Zubarev, R. A. Electron Capture Dissociation and Other Ion-Electron Fragmentation Reactions. In Principles of Mass Spectrometry Applied to Biomolecules, Lifshitz, C.; Laskin, J., Eds.; John Wiley and Sons Inc.: Hoboken, NJ, 2006; p. 475-518.

4. Syka, J. E. P.; Coon, J. J.; Schroeder, M. J.; Shabanowitz, J.; Hunt, D. F Peptide and Protein Sequence Analysis by Electron Transfer Dissocia- 
tion Mass Spectrometry. Proc. Nat. Acad. Sci. U.S.A. 2004, 101(26), 9528-9533.

5. Coon, J. J.; Ueberheide, B.; Syka, J. E. P.; Dryhurst, D. D.; Ausio, J.; Shabanowitz, J.; Hunt, D. F. Protein Identification Using Sequential Ion/Ion Reactions and Tandem Mass Spectrometry. Proc. Nat. Acad. Sci. U.S.A. 2005, 102(27), 9463-9468.

6. Budnik, B. A.; Nielsen, M. L.; Olsen, J. V.; Haselmann, K. F.; Horth, P.; Haehnel, W.; Zubarev, R. A. Can Relative Cleavage Frequencies in Peptides Provide Additional Sequence Information? Int. J. Mass Spectrom. 2002, 219(1), 283-294.

7. Breuker, K.; Oh, H. B.; Lin, C.; Carpenter, B. K.; McLafferty, F. W. Nonergodic and Conformational Control of the Electron Capture Dissociation of Protein Cations. Proc. Nat. Acad. Sci. U.S.A. 2004, 101(39), 14011-14016.

8. Adams, C. M.; Kjeldsen, F.; Zubarev, R. A.; Budnik, B. A.; Haselmann, K. F. Electron Capture Dissociation Distinguishes a Single D-Amino Acid in a Protein and Probes the Tertiary Structure. J. Am. Soc. Mass Spectrom. 2004, 15(7), 1087-1098.

9. Oh, H.; Breuker, K.; Sze, S. K.; Ge, Y.; Carpenter, B. K.; McLafferty, F. W. Secondary and Tertiary Structures of Gaseous Protein Ions Characterized by Electron Capture Dissociation Mass Spectrometry and Photofragment Spectroscopy. Proc. Nat. Acad. Sci. U.S.A. 2002, 99(25), 1586315868

10. Lin, C.; Cournoyer, J. J.; O'Connor, P. B. Probing the Gas-Phase Folding Kinetics of Peptide Ions by IR Activated DR-ECD. J. Am. Soc. Mass Spectrom. 2008, 19(6), 780-789.

11. Savitski, M. M.; Kjeldsen, F.; Nielsen, M. L.; Zubarev, R. A. Complementary Sequence Preferences of Electron-Capture Dissociation and Vibrational Excitation in Fragmentation of Polypeptide Polycations. Angew. Chem. Int. Ed. 2006, 45(32), 5301-5303.

12. Ben Hamidane, H.; He, H.; Tsybin, O. Y.; Emmett, M. R.; Hendrickson, C. L.; Marshall, A. G.; Tsybin, Y. O. Periodic Sequence Distribution of Product Ion Abundances in Electron Capture Dissociation of Amphipathic Peptides and Proteins. J. Am. Soc. Mass Spectrom. 2009, 20(6), $1182-1192$.

13. Polfer, N. C.; Haselmann, K. F.; Langridge-Smith, P. R. R.; Barran, P. E. Structural Investigation of Naturally Occurring Peptides by Electron Capture Dissociation and AMBER Force Field Modeling. Mol. Phys. 2005, 103(11/12), 1481-1489.

14. Patriksson, A.; Adams, C.; Kjeldsen, F.; Raber, J.; van der Spoel, D.; Zubarev, R. A. Prediction of $\mathrm{N}-\mathrm{C}_{\alpha}$ Bond Cleavage Frequencies in Electron Capture Dissociation of Trp-Cage Dications by Force-Field Molecular Dynamics Simulations. Int. J. Mass Spectrom. 2006, 248(3), $124-135$.

15. Tsybin, Y. O.; Ben Hamidane, H.; Vorobyev, A.; Wodrich, M.; Corminboeuf, C. Peptide Conformation Selectivity in Electron Capture and Transfer Dissociation. Proceedings of the 57th ASMS Conference on Mass Spectrometry and Allied Topics; Philadelphia, PA, June 2009.

16. Prell, J. S.; O’Brien, J. T.; Holm, A. I. S.; Leib, R. D.; Donald, W. A.; Williams, E. R. Electron Capture by a Hydrated Gaseous Peptide: Effects of Water on Fragmentation and Molecular Survival. J. Am. Chem. Soc. 2008, 130(38), 12680-12689.

17. Ben Hamidane, H.; Chiappe, D.; Hartmer, R.; Vorobyev, A.; Moniatte, M.; Tsybin, Y. O. Electron Capture and Transfer Dissociation: Peptide Structure Analysis at Different Ion Internal Energy Levels. J. Am. Soc. Mass Spectrom. 2009, 20(4), 567-575.

18. Tsybin, Y. O.; Ben Hamidane, H.; Vorobyev, A.; Albrieux, F.; Antoine, R.; Calvo, F.; Chirot, F.; Lemoine, J.; Dugourd, P. A Systematic Approach to Complementary Electron Capture Dissociation and Ion Mobility in Peptide Conformation Studies, unpublished.

19. Parks, B. A.; Jiang, L.; Thomas, P. M.; Wenger, C. D.; Roth, M. J.; Boyne, M. T.; Burke, P. V.; Kwast, K. E.; Kelleher, N. L. Top-Down Proteomics on a Chromatographic Time Scale Using Linear Ion Trap Fourier Transform Hybrid Mass Spectrometers. Anal. Chem. 2007, 79, 79847991.

20. Schaub, T. M.; Hendrickson, C. L.; Horning, S.; Quinn, J. P.; Senko, M. W.; Marshall, A. G. High-Performance Mass Spectrometry: Fourier Transform Ion Cyclotron Resonance at 14.5 Tesla. Anal. Chem. 2008, 80(11), 3985-3990.

21. Tsybin, Y. O.; Quinn, J. P.; Tsybin, O. Y.; Hendrickson, C. L.; Marshall, A. G. Electron Capture Dissociation Implementation Progress in Fourier Transform Ion Cyclotron Resonance Mass Spectrometry. J. Am. Soc. Mass Spectrom. 2008, 19(6), 762-771.

22. Tsybin, Y. O.; Hendrickson, C. L.; Beu, S. C.; Marshall, A. G. Impact of Ion Magnetron Motion on Electron Capture Dissociation Fourier Transform Ion Cyclotron Resonance Mass Spectrometry. Int. J. Mass Spectrom. 2006, 255, 144-149.
23. Tsybin, Y. O.; He, H.; Emmett, M. R.; Hendrickson, C. L.; Marshall, A. G. Ion Activation in Electron Capture Dissociation to Distinguish Between N-Terminal and C-Terminal Productions. Anal. Chem. 2007, 79, 75967602.

24. Kjeldsen, F.; Savitski, M. M.; Adams, C. M.; Zubarev, R. A. Determination of the Location of Positive Charges in Gas-Phase Polypeptide Polycations by Tandem Mass Spectrometry. Int. J. Mass Spectrom. 2006, 252(3), 204-212.

25. Sun, Q. Y.; Nelson, H.; Ly, T.; Stoltz, B. M.; Julian, R. R. Side Chain Chemistry Mediates Backbone Fragmentation in Hydrogen Deficient Peptide Radicals. J. Proteome Res. 2009, 8(2), 958-966.

26. Pazos, M.; Andersen, M. L.; Skibsted, L. H. Amino Acid and Protein Scavenging of Radicals Generated by Iron/Hydroperoxide System: An Electron Spin Resonance Spin Trapping Study. J. Agric. Food Chem. 2006, 54(26), 10215-10221.

27. Grantham, R. Amino-Acid Difference Formula to Help Explain Protein Evolution. Science 1974, 185(4154), 862-864.

28. Creese, A. J.; Cooper, H. J. The Effect of Phosphorylation on the Electron Capture Dissociation of Peptide Ions. J. Am. Soc. Mass Spectrom. 2008, 19(9), 1263-1274.

29. Joly, L.; Antoine, R.; Allouche, A. R.; Dugourd, P. Formation and Spectroscopy of a Tryptophan Radical Containing Peptide in the Gas Phase. J. Am. Chem. Soc. 2008, 130(42), 13832-13833.

30. Belyayev, M. A.; Cournoyer, J. J.; Lin, C.; O'Connor, P. B. The Effect of Radical Trap Moieties on Electron Capture Dissociation Spectra of Substance P. J. Am. Soc. Mass Spectrom. 2006, 17(10), 1428-1436.

31. Gasteiger, E.; Hoogland, C.: Gattiker, A.; Duvaud, S.; Wilkins, M. R. Appel, R. D.; Bairoch, A. Protein Identification and Analysis Tools on the ExPASy Server. In The Proteomics Protocols Handbook (Methods in Molecular Biology), Walker, J. M., Ed.; Humana Press: Totowa, NJ, 2005; pp 571-607.

32. Hessa, T.; Kim, H.; Bihlmaier, K.; Lundin, C.; Boekel, J.; Andersson, H. Nilsson, I.; White, S. H.; von Heijne, G. Recognition of Transmembrane Helices by the Endoplasmic Reticulum Translocon. Nature 2005, 433(7024), 377-381.

33. Kyte, J.; Doolittle, R. F. A Simple Method for Displaying the Hydropathic Character of a Protein. J. Mol. Biol. 1982, 157(1), 105-132.

34. Black, S. D.; Mould, D. R. Development of Hydrophobicity Parameters to Analyze Proteins Which Bear Posttranslational or Cotranslational Modifications. Anal. Biochem. 1991, 193(1), 72-82.

35. Zimmerman, J.; Eliezer, N.; Simha, R. Characterization of Amino Acid Sequences in Proteins by Statistical Methods. J. Theor. Biol. 1968, 21(2), 170-201.

36. Deleage, G.; Roux, B. An Algorithm for Protein Secondary Structure Prediction Based on Class Prediction. Protein Eng. 1987, 1(4), 289-294.

37. Zhao, G.; London, E. An Amino Acid "Transmembrane Tendency" Scale That Approaches the Theoretical Limit to Accuracy for Prediction of Transmembrane Helices: Relationship to Biological Hydrophobicity. Protein Sci. 2006, 15(8), 1987-2001.

38. Bohorquez, H. J.; Obregon, M.; Cardenas, C.; Llanos, E.; Suarez, C. Villaveces, J. L.; Patarroyo, M. E. Electronic Energy and Multipolar Moments Characterize Amino Acid Side Chains into Chemically Related Groups. J. Phys. Chem. A 2003, 107(47), 10090-10097.

39. Bhaskaran, R.; Ponnuswamy, P. K. Positional Flexibilities of AminoAcid Residues in Globular-Proteins. Int. J. Pept. Protein Res. 1988, 32(4), 241-255.

40. Charton, M.; Charton, B. I. The Dependence of the Chou-Fasman Parameters on Amino-Acid Side-Chain Structure. J. Theor. Biol. 1983 102(1), 121-134.

41. Dwyer, D. S. Electronic Properties of the Amino Acid Side Chains Contribute to the Structural Preferences in Protein Folding. J. Biomol. Struct. Dynamics 2001, 18(6), 881-892.

42. Cowan, R.; Whittaker, R. G. Hydrophobicity Indices for Amino Acid Residues as Determined by HPLC. Pept. Res. 1990, 3, 75-80.

43. Pouthier, V.; Tsybin, Y. O. Amide-I Relaxation-Induced Hydrogen Bond Distortion: An Intermediate in Electron Capture Dissociation Mass Spectrometry of $\alpha$-Helical Peptides? J. Chem. Phys. 2008, 129, 95-106.

44. Schlag, E. W.; Sheu, S. Y.; Yang, D. Y.; Selzle, H. L.; Lin, S. H. Distal Charge Transport in Peptides. Angew. Chem. Int. Ed. 2007, 46(18), 3196-3210.

45. Sheu, S. Y.; Schlag, E. W. Protein Charge Transport in Gas Phase. Int. J. Mass Spectrom. 2002, 219(1), 73-77.

46. Hennig, D. Energy Transport in $\alpha$-Helical Protein Models: One-Strand Versus Three-Strand Systems. Phys. Rev. B 2002, 65(17).

47. Remacle, F.; Levine, R. D.; Schlag, E. W.; Weinkauf, R. Electronic Control of Site Selective Reactivity: A Model Combining Charge Migration and Dissociation. J. Phys. Chem. A 1999, 103(49), 10149-10158. 